\title{
Rise velocity of a spherical cap bubble
}

\author{
By DANIEL D. JOSEPH \\ University of Minnesota, Aerospace Engineering and Mechanics, 110 Union St. SE, Minneapolis, \\ MN 55455, USA
}

(Received 23 October 2002 and in revised form 26 February 2003)

The theory of viscous potential flow is applied to the problem of finding the rise velocity $U$ of a spherical cap bubble (see Davies \& Taylor 1950; Batchelor 1967). The rise velocity is given by

$$
\frac{U}{\sqrt{g D}}=-\frac{8}{3} \frac{v(1+8 s)}{\sqrt{g D^{3}}}+\frac{\sqrt{2}}{3}\left[1-2 s-\frac{16 s \sigma}{\rho g D^{2}}+\frac{32 v^{2}}{g D^{3}}(1+8 s)^{2}\right]^{1 / 2},
$$

where $R=D / 2$ is the radius of the cap, $\rho$ and $v$ are the density and kinematic viscosity of the liquid, $\sigma$ is surface tension, $r(\theta)=R\left(1+s \theta^{2}\right)$ and $s=r^{\prime \prime}(0) / D$ is the deviation of the free surface from perfect sphericity $r(\theta)=R$ near the stagnation point $\theta=0$. The bubble nose is more pointed when $s<0$ and blunted when $s>0$. A more pointed bubble increases the rise velocity; the blunter bubble rises slower. The Davies \& Taylor (1950) result arises when $s$ and $v$ vanish; if $s$ alone is zero,

$$
\frac{U}{\sqrt{g D}}=-\frac{8}{3} \frac{v}{\sqrt{g D^{3}}}+\frac{\sqrt{2}}{3}\left[1+\frac{32 v^{2}}{g D^{3}}\right]^{1 / 2},
$$

showing that viscosity slows the rise velocity. This equation gives rise to a hyperbolic drag law

$$
C_{D}=6+32 / R_{e}
$$

which agrees with data on the rise velocity of spherical cap bubbles given by Bhaga \& Weber (1981).

\section{Analysis}

Potential flow is a solution of the Navier-Stokes equations; it satisfies all of the usual equations for inviscid potential flow, like Bernoulli's equation, the CauchyLagrange equation, etc., except that the viscous stresses do not in general vanish. Viscous potential flows give rise to excellent physical results for flows with interfaces; for such flows the viscosity enters the analysis explicitly through the normal stress balance. The Rayleigh-Plesset bubble is an exact viscous potential flow analysis of the Navier-Stokes equations; this solution works perfectly for all viscous liquids; it is not an asymptotic result. Viscous potential flow analysis of problems for RayleighTaylor instability (Joseph, Belanger \& Beavers 1999; Joseph, Beavers \& Funada 2002), for capillary instability (Funada \& Joseph 2001) and Kelvin-Helmholtz instability (Funada \& Joseph 2002) gives rise to solutions dependent strongly on viscosity and closely approximate solutions of the problem in which potential flow is not assumed. Viscous potential flow has zero vorticity and it will certainly fail when vorticity is important. 


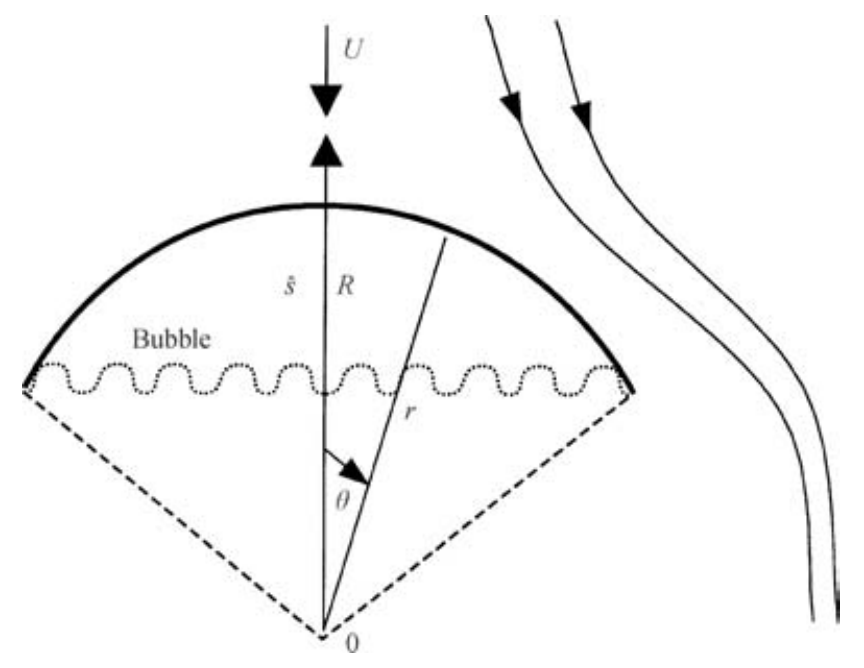

FIGURE 1. Spherical cap bubble. The rising bubble is viewed in a frame in which the bubble is stationary. The origin of $z$ increasing is at the stagnation point $\hat{s}$. The surface of the cap is given by $z=-h(r, \theta)=-(R-r(\theta) \cos \theta)$. The cap is strictly spherical if $r(\theta)=R$ is constant.

The spherical cap bubble (figure 1) arises in the motion of large gas bubbles which take a lenticular shape. The analysis of the rise velocity of these bubbles which was given by Davies \& Taylor (1950) is unusual since it is not computed from a balance of the drag and buoyant weight as it is for spherical gas bubbles (Levich 1949; Moore 1959, 1963; Taylor \& Acrivos 1964; Miksis, Vanden-Broeck \& Keller 1982; Ryskin \& Leal 1984). Batchelor (1967) notes that "The remarkable feature of (the Davies-Taylor analysis) is that the speed of movement of the bubble is derived in terms of the bubble shape without any need for consideration of the mechanism of the retarding force which balances the effect of the buoyancy force on a bubble in steady motion".

In the analysis which follows, I extend the analysis of Davies \& Taylor which is based on inviscid potential flow to viscous potential flow and I show that surface tension enters the formula for the rise velocity only when the axisymmetric bubble is not spherical.

The velocity field on the gas bubble and the liquid is derived from a potential $\boldsymbol{u}=\nabla \phi, \nabla^{2}=0$. The velocity at $z=\infty$ is $-U$ (against $z$ ) and $\boldsymbol{g}=-\boldsymbol{e}_{z} g$. For steady flow

$$
\rho \boldsymbol{u} \cdot \nabla \boldsymbol{u}=-\nabla p-\rho \boldsymbol{e}_{z} g=-\nabla \Gamma
$$

where

$$
\Gamma=p+\rho g z
$$

Equation (1.1) may be integrated, since $\boldsymbol{u} \cdot \nabla \boldsymbol{u}=\nabla|\boldsymbol{u}|^{2} / 2$, giving rise to a Bernoulli function in the liquid

$$
\frac{\rho|\boldsymbol{u}|^{2}}{2}+\Gamma=\frac{\rho U^{2}}{2}
$$


and in the gas

$$
\frac{\rho_{G}|\boldsymbol{u}|^{2}}{2}+\Gamma_{G}=C_{G},
$$

where $C_{G}$ is an unknown constant.

We turn next to the normal stress balance

$$
-\llbracket p \rrbracket+2 \llbracket \mu \boldsymbol{n} \cdot \boldsymbol{D}[\boldsymbol{u}] \rrbracket \cdot \boldsymbol{n}+\frac{2 \sigma}{r(\theta)}=0,
$$

where

$$
\llbracket \cdot \rrbracket=(\cdot)_{G}-(\cdot)_{L}
$$

is evaluated on the free surface $r(\theta)=R\left(1+s \theta^{2}\right), \sigma$ is surface tension, $\mu$ is viscosity and

$$
\boldsymbol{n} \cdot \boldsymbol{D}[\boldsymbol{u}] \cdot \boldsymbol{n}=\frac{\partial u_{n}}{\partial n}
$$

is the normal component of the rate of strain. Using (1.1), (1.4) and (1.5) we obtain

$$
-\llbracket \Gamma \rrbracket-\llbracket \rho \rrbracket g h+2\left[\left[\mu \frac{\partial u_{n}}{\partial n}\right]\right]+\frac{2 \sigma}{r}=0
$$

where $-h$ is the value of $z$ on the free surface.

Following Davies \& Taylor (1950), we assume that $\boldsymbol{u}$ may be approximated near the stagnation point on the bubble, which is nearly spherical, by the potential for the sphere; thus

$$
\phi=-U r \cos \theta\left(1+\frac{R^{3}}{2 r^{3}}\right)
$$

for the liquid. The form of $\phi$ in the gas will not be needed. From (1.7) we compute

$$
\begin{gathered}
u_{r}=\frac{\partial \phi}{\partial r}=-U\left(1-\frac{R^{3}}{r^{3}}\right) \cos \theta, \\
u_{\theta}=\frac{1}{r} \frac{\partial \phi}{\partial \theta}=U \sin \theta\left(1+\frac{R^{3}}{2 r^{3}}\right), \\
\frac{\partial u_{n}}{\partial n}=\frac{\partial u_{r}}{\partial r}=-\frac{3 U R^{3}}{r^{4}} \cos \theta .
\end{gathered}
$$

The functions (1.8), (1.9) and (1.10) enter into the normal stress balance at $r=$ $R\left(1+s \theta^{2}\right)$. This balance is to be satisfied near the stagnation point, for small $\theta$, neglecting terms that go to zero faster than $\theta^{2}$. At the free surface,

$$
\begin{gathered}
u_{r}=-U\left\{1-\frac{1}{\left(1-s \theta^{2}\right)^{3}}\right\}=-3 U s \theta^{2}, \quad u_{\theta}=\frac{3}{2} U \theta, \\
\frac{\partial u_{n}}{\partial n}=-\frac{3 U\left(1-\frac{1}{2} \theta^{2}\right)}{R\left(1+s \theta^{2}\right)^{4}}=-\frac{3 U}{R}\left\{1-\left(4 s+\frac{1}{2}\right) \theta^{2}\right\},
\end{gathered}
$$




$$
\begin{gathered}
u_{r}^{2}=0, \\
u_{\theta}^{2}=\frac{9}{4} U^{2} \theta^{2}, \\
h=R-r \cos \theta=R-R\left(1+s \theta^{2}\right)\left(1-\frac{1}{2} \theta^{2}\right)=R\left(\frac{1}{2}-s\right) \theta^{2} .
\end{gathered}
$$

The motion of the gas in the bubble is not known but it enters into (1.6) as the coefficient of $\rho_{G}$ and $\mu_{G}$, which are small relative to the corresponding liquid terms. Evaluating (1.2) and (1.3) on the free surface, with gas motion zero, we obtain

$$
\begin{gathered}
\Gamma=-\frac{9}{8} \rho U^{2} \theta^{2}+\rho \frac{U^{2}}{2}, \\
\Gamma_{G}=C_{G} .
\end{gathered}
$$

Using (1.11) to (1.16), we may rewrite (1.6) as

$$
\begin{aligned}
0= & -C_{G}+\Gamma+\rho g h-2 \mu \frac{\partial u_{r}}{\partial r}+\frac{2 \sigma}{r} \\
= & -C_{G}+\frac{\rho U^{2}}{2}-\frac{9}{8} \rho U^{2} \theta^{2}+\rho g R\left(\frac{1}{2}-s\right) \theta^{2} \\
& +\frac{6 \mu U}{R}\left\{1-\left(4 s+\frac{1}{2}\right) \theta^{2}\right\}+\frac{2 \sigma}{R}\left(1-s \theta^{2}\right) .
\end{aligned}
$$

The constant terms vanish:

$$
C_{G}=\frac{\rho U^{2}}{2}+\frac{6 \mu U}{R}+\frac{2 \sigma}{R} .
$$

The coefficient of $\theta^{2}$ also vanishes:

$$
\frac{9}{8} \rho U^{2}+\frac{3 \mu U}{R}+\frac{24 \mu U s}{R}=\rho g \frac{R}{2}-s\left(\rho g R+\frac{2 \sigma}{R}\right) .
$$

Surface tension, which balances the static pressure difference in a sphere or spherical cap, enters the formula for the velocity only when the axisymmetric cap is not spherical. When the spherical cap is perfectly spherical, as in the case treated by Davies \& Taylor (1950), $s=0$ and

$$
U=-\frac{4}{3} \frac{v}{R}+\sqrt{\frac{4}{9} g R+\frac{16}{9} \frac{v^{2}}{R^{2}}} .
$$

Equation (1.20) shows that the viscosity slows the rise velocity; when the viscosity is much larger than gravity

$$
U=\frac{1}{6} \frac{g R^{2}}{v},
$$

which is the velocity of a rising sphere computed by Moore (1959) from viscous potential flow balancing the drag with the buoyant weight.

The general solution of (1.19) with $D=2 R$ is

$$
\frac{U}{\sqrt{g D}}=-\frac{8}{3} \frac{v(1+8 s)}{\sqrt{g D^{3}}}+\frac{\sqrt{2}}{3}\left[1-2 s-\frac{16 s \sigma}{\rho g D^{2}}+\frac{32 v^{2}}{g D^{3}}(1+8 s)^{2}\right]^{1 / 2} .
$$


It is convenient to write (1.22) in a dimensionless form:

$$
F r=-\frac{8(1+8 s)}{3 \mathfrak{R}_{G}}+\frac{\sqrt{2}}{3}\left[1-2 s-\frac{16 s}{E \ddot{o}}+\frac{32}{\mathfrak{R}_{G}^{2}}(1+8 s)^{2}\right]^{1 / 2},
$$

where

$$
\begin{aligned}
F r & =\frac{U}{\sqrt{g D}} \quad \text { Froude number, } \\
\mathfrak{R}_{G}=\frac{\sqrt{g D^{3}}}{\nu} & \text { gravity Reynolds number, } \\
E \ddot{o}=\frac{\rho g D^{2}}{\sigma} & \text { Eötvös number. }
\end{aligned}
$$

These three parameters are the only ones that enter into correlations for the rise velocity of long bubbles in round pipes (Wallis 1969; Vania et al. 2002).

It is of interest to consider different effects entering into the formula for the rise velocity $U$ given by (1.22). The Davies \& Taylor formula

$$
U=\frac{\sqrt{2}}{3} \sqrt{g D}
$$

arises from (1.22) when $v=\sigma=s=0$. Recall that $s=r^{\prime \prime}(0) / D$ represents the difference from an undeformed spherical cap. When $s=0$ the cap is exactly spherical; when $s<0$ the nose of the cap is more pointed than the spherical cap and when $s>0$ the nose is blunter than a sphere. The Davies-Taylor formula (1.24) arising from (1.22) in the asymptotic limit for large values of $\mathfrak{R}$ and $E \ddot{o}$

$$
U=\frac{\sqrt{2}}{3}(1-2 s) \sqrt{g D}
$$

holds only when $s=0$.

Unfortunately the analysis does not give the value of $s$; the shape of the nose is given when $U$ is known, or if $s$ is known then $U$ is predicted. A more satisfying result would need to relax the assumption that the velocity potential (1.7) does not change when the spherical cap is not exactly spherical. This kind of perturbation analysis requires global data and is well beyond what can at present be obtained by analysis.

\section{Experiments}

A review of experiments on the rise of spherical cap bubbles prior to 1973 together with an excellent collection of photographs can be found in the paper by Wegener \& Parlange (1973). Reviews treating rising bubbles of all types were presented by Harper (1972) and Bhaga \& Webber (1981).

The comparison of the prediction (1.23) of the rise velocity of a sperical cap bubble arising from the application of viscous potential flow is unambiguous when the deviation $s$ from sphericity vanishes. There are two cases in which $s=0$ : according to the analysis of Davies \& Taylor (1950), the spherical cap which arises for large bubbles is one case; the other case includes the rise of small bubbles, or bubbles with large surface tension, which was considered by Levich (1949) and Moore $(1959,1963)$. In the first the sphericity arises from dynamics alone and the effects of surface tension on the spherical cap is negligible. In the case of small bubbles, or bubbles moving very slowly, surface tension can be important in keeping the bubble spherical, but the effect then of surface tension is absorbed totally by the pressure drop $\llbracket p \rrbracket=2 \sigma / R$, as 


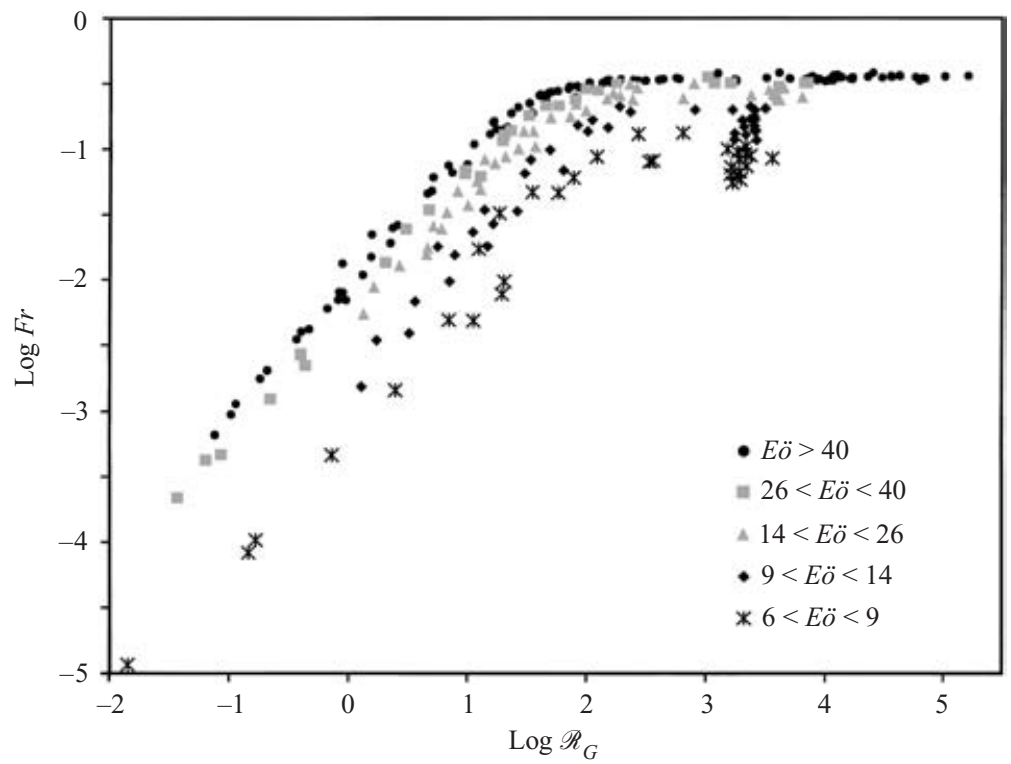

Figure 2. (After Vania et al. 2002). Rise velocity $F r$ vs. $\mathfrak{R}_{G}$ for different Eötvös numbers for all published experiments on the rise velocity of Taylor bubbles in round pipes. The rise velocity is independent of $E \ddot{o}$ when $E \ddot{o}>40$.

in (1.18), and does not enter the dynamics. The effects of surface tension on the rise velocity are associated with the deviation from sphericity; it is a shape effect since the net force and moment on a smooth bubble due to surface forces are zero (Hesla, Huang \& Joseph 1993).

An efficient description of the effects affecting the deformation and rise velocity of gas bubbles in stagnant liquids can be carried out in terms of dimensionless parameters. The formula (1.23) expresses a functional relation between three parameters: the Froude number, the gravity Reynolds number $\mathfrak{R}_{G}$ and the Eötvös number $E \ddot{o}$; these three parameters completely describe the rise velocity of Taylor bubbles, which are long gas bubbles capped by a spherical cap rising in tubes filled with stagnant liquid which were discussed in the Davies-Taylor paper. Vania et al. (2002) correlated all the published data, 262 experiments, on the rise velocity, $F r$, of Taylor bubbles in round pipes with a highly accurate rational fraction of power of the parameters $\mathfrak{R}_{G}$ and $E \ddot{o}$; a graph of the data processed by them is shown in figure 2 .

Other parameters are frequently used for the description of the rise velocity; these are

$$
\left.\begin{array}{ll}
C_{D}=\frac{4}{3} \frac{g d_{e}}{U^{2}} & \text { drag coefficient, } \\
R_{e}=\frac{U d_{e}}{v} & \text { Reynolds number, } \\
W=\frac{\rho U^{2} d_{e}}{\sigma} & \text { Weber number, } \\
M=\frac{g \mu^{4}}{\rho \sigma^{3}} & \text { Morton number. }
\end{array}\right\}
$$




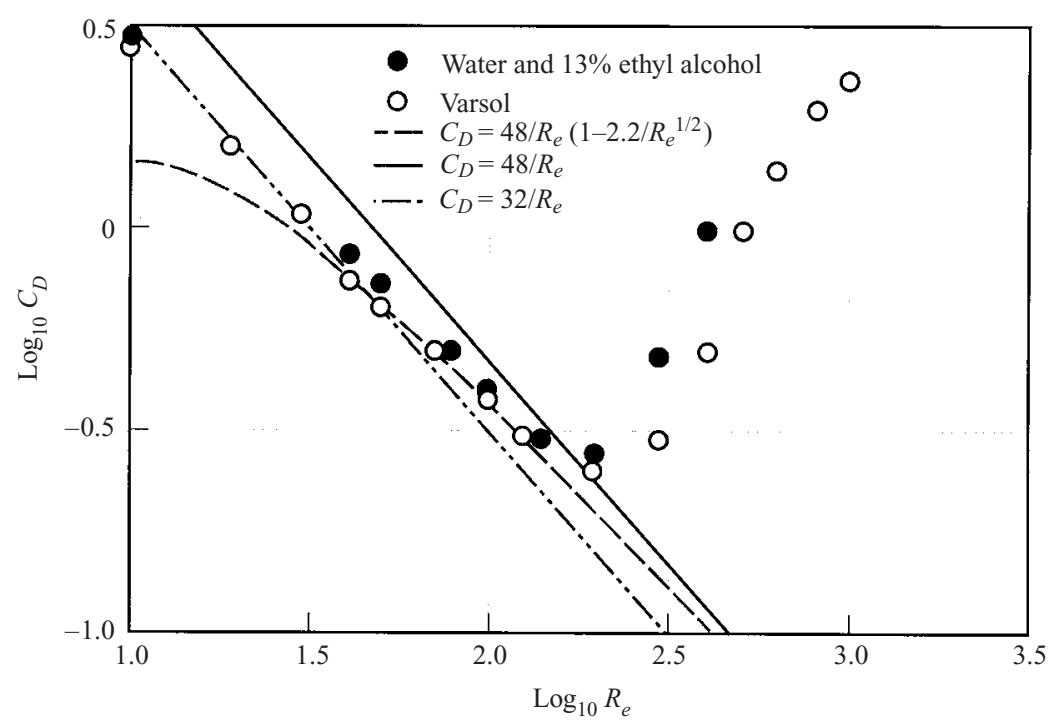

Figure 3. (After Batchelor 1967). The drag coefficient of gas bubbles rising through liquids. The points for two particular liquids are taken from experimental curves given by Haberman $\&$ Morton (1953). The line $C_{D}=32 / R_{e}$ was added by me. The formulae use effective diameters $d_{e}=D$ where $R=D / 2$ is the radius of the sphere.

The choice of effective diameter is important; two choices are made here: $d_{e}=D$ where $d_{e}$ is the sphere diameter or the diameter of the spherical cap, $d_{e}=\bar{d}$ where $\bar{d}$ is volume equivalent diameter defined by $V=\frac{1}{6} \pi \bar{d}^{3}$, and Reynolds numbers by $R_{e}=u D / \nu, \bar{R}_{e}=u \bar{d} / \nu$ based on $D$ and $\bar{d}$. Any pair of independent dimensionless parameters determine all the others in steady flow; as in (1.23), a dimensionless parameter involving $U$ may be expressed in terms of two other independent parameters.

It is convenient to compare the theory developed here with experiments in which the surface tension, or parameters in which the surface tension is a factor, affect the rise velocity and those for which these parameters are not important; for example, according to figure $2, F r$ is a function of $\mathfrak{R}_{G}$ alone when $E \ddot{o}>40$.

In the case of a spherical cap bubble, limitations on the bubble size arise from several sources and restrict the values of the parameters which may be observed. Grace, Wairegi \& Brophy (1978) reported the maximum volume of air in bubbles that remain intact in five different liquids in a wide tank but did not identify which of the five were spherical cap. Batchelor (1987) looked at these data in terms of a stability theory which limits the maximum size bubbles. A numerical study by Bolton-Stone, Robinson \& Blake (1995) suggests that spherical cap bubbles arise only when the Eötvös number based on an equivalent spherical radius is less than about 32. For higher values of $E \ddot{o}$ an unstable toroidal bubble is formed before breakup.

Figure 3 was presented in Batchelor's (1967) book. He compared experiments with drag laws $C_{D}=48 / R_{e}$ computed by Levich (1949) using the dissipation approximation and Moore's $(1959) C_{D}=\left(48 / R_{e}\right)\left(1-2.2 / R_{e}^{1 / 2}\right)$ correction of this law. Moore tried to correct the pressure jump across the spherical bubble surface for effects of a vorticity boundary layer. Kang \& Leal (1988) showed that Moore's result is incomplete because it does not include displacement thickness effects; they found $48 / R$ at leading order 


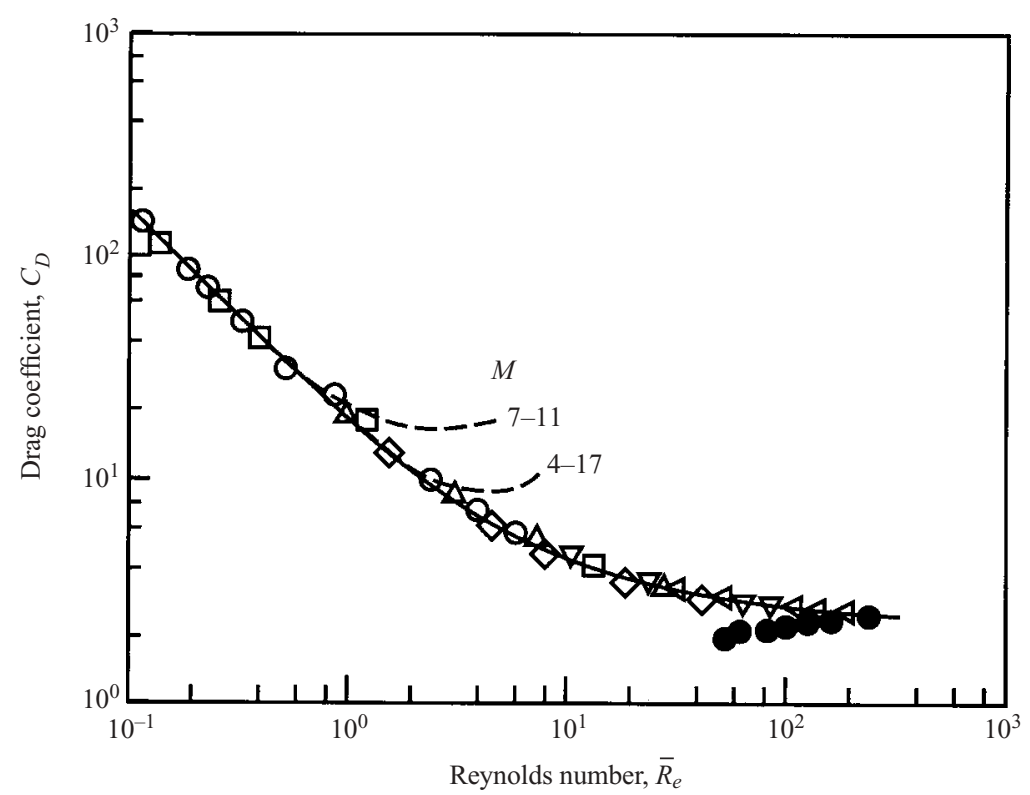

FIGURE 4. (After figure 7 in Bhaga $\&$ Weber 1981.) $\bar{C}_{D}\left(=4 g \bar{d} / U^{2}\right)$ vs. $\bar{R}_{e}(=U \bar{d} / v)$. Taylor \& Acrivos $1964(--) ; \bar{C}_{D}$ given by $(2.2)(-) ; M=1.64 \times 10^{-3}(\bullet)$.

using a general relationship between the viscous pressure correction and the vorticity distribution for a spherical bubble in an arbitrary axisymmetric flow. The values of $C_{D}$ given by Stoke's flow, viscous potential flow and the dissipation are $16 / R_{e}, 32 / R_{e}$ and $48 / R_{e}$, respectively.

It seems to me that the experiments in figure 3 are not convincing evidence for any of the three drag laws just mentioned. The deviation from a linear law, say $32 / R_{e}$ rather than $48 / R_{e}$, might be interpreted in terms of changes in drag due to bubble deformation rather than any effect of vorticity on the pressure jump. This interpretation is consistent with the experiments and with the analysis of Taylor \& Acrivos (1964) and Ryskin \& Leal (1984), who showed that bubbles resembling spherical caps arise when the Weber number is large, as in the points past the minimum, and perhaps before the minimum $C_{D}$ in figure 3 . Evidently, it is not very easy to do experiments in which an $48 / R_{e}$ drag law is actually realized.

The data shown in figure 3 are quite different from the data presented in figure 4. The data in figure 3 do not apply to the spherical cap bubble that is considered in this paper. The large- $R_{e}$ limit leading in figure 4 to the Davies-Taylor limit is in excellent agreement with experiments and is not likely to be greatly improved by a boundary layer analysis, even if it could be carried out.

Harper (1972) makes a distinction between high-and low-Morton-number $(M)$ liquids. He characterizes the high- $M$ liquids as those for which $C_{D}$ decreases monotonically with $\bar{R}_{e}$ as in figure 4 , and the low- $M$ liquids as those for which $C_{D}$ vs. $\bar{R}_{e}$ has a minimum as in figure 3 . Bhaga $\&$ Weber (1981) identify this critical value as $M=4 \times 10^{-3}$; a low- $M$ liquid response is identified by the solid circle data points in figure 4.

The monotonic curve through the high- $M$ open symbol points in figure 4 is described by an empirical formula

$$
\bar{C}_{D}=\left[(2.67)^{0.9}+\left(16 / \bar{R}_{e}\right)^{0.9}\right]^{1 / 0.9}
$$




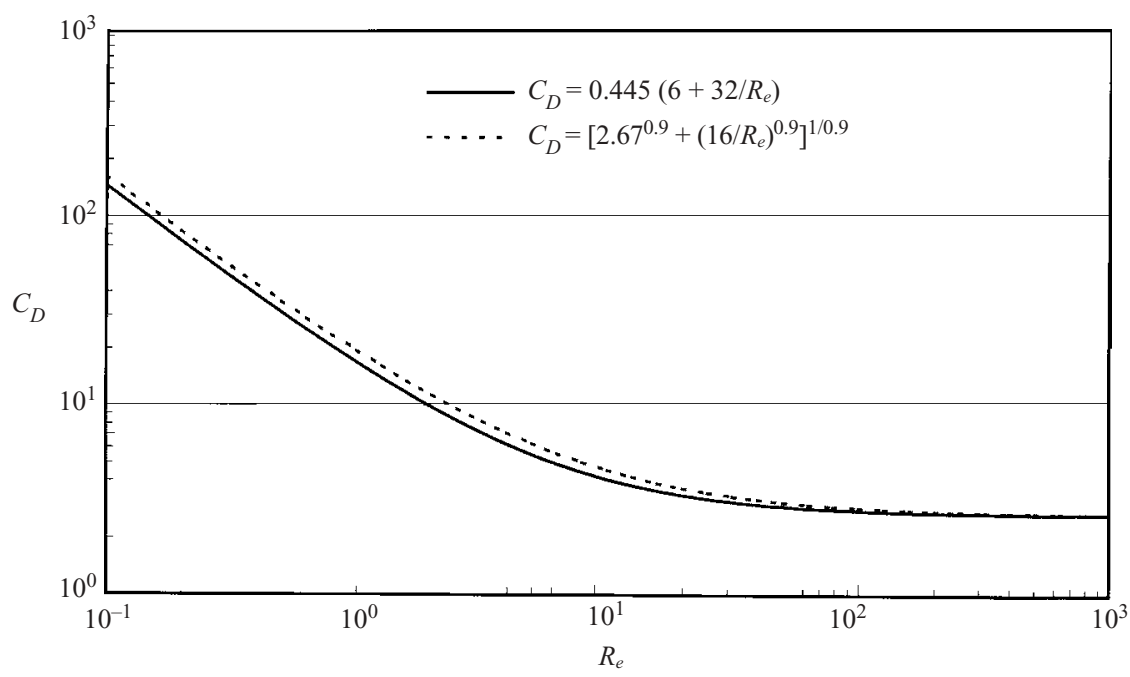

Figure 5. Comparison of the empirical drag law (2.2) with the theoretical drag law (2.6) scaled by the factor 0.445 required to match the data in figure 4 with the experiments of Davies \& Taylor (1950) at large $R_{e}$.

in which $\bar{C}_{D}$ is determined by $\bar{R}_{e}$ alone. The shapes of the bubbles change from spheres to spherical cap bubbles as $\bar{R}_{e}$ increases; $\bar{C}_{D}$ and $\bar{R}_{e}$ are defined here using the volume equivalent diameter $\bar{d}$, which decreases from the sphere diameter $D$ at low $\bar{R}_{e}$ to a value $\bar{d}<D$ for a spherical cap bubble which can be estimated from the results of Davies \& Taylor (1950) and those given here.

Consider the case in which the deviation from sphericity $s=0$. In this case equation (1.20) holds. We may rewrite this equation as a drag relation

$$
C_{D}=6+32 / R_{e},
$$

where $C_{D}=\frac{4}{3}\left(g D / u^{3}\right), R_{e}=U D / v$ and $D=2 R$ is the diameter of the spherical cap. The large- $R_{e}$ limit of (2.3), $C_{D}=6$ is the value of drag coefficient which was established in the brilliant experiments by Davies \& Taylor (1950). The asymptotic large- $\bar{R}_{e}$ limit of (2.2) is $\bar{C}_{D}=2.67$. These two limits should be the same, hence

$$
\frac{\bar{C}_{D}}{C_{D}}=\frac{2.67}{6}=\frac{\bar{d}}{D} .
$$

If this limit is a spherical cap bubble the volume equivalent diameter is

$$
\bar{d}=0.445 D
$$

for the spherical cap diameter $d=2 R$. The ratio of the volume of the spherical cap bubble to the volume of a sphere of radius $R=D / 2$ is

$$
\bar{V}=\frac{\overline{d^{3}} V}{D^{3}}=0.0881 \text {. }
$$

The volume of the spherical cap bubble in this computation is a little less than $1 / 10$ the value of the volume of the sphere from which it is cut.

In comparing (2.2) and (2.3) we must convert $\bar{R}_{e}$ to $R_{e}$ and though this cannot be done generally, it can be done empirically for the limiting case of large $R_{e}$ for which $R_{e}=0.445 R_{e}$. In figure 5 we compare the empirical relation (2.2) to a rescaled plot 
of (2.3)

$$
\tilde{C}_{D}=0.445\left(6+\frac{32}{R_{e}}\right) .
$$

The agreement between (2.6) and (2.2) is spectacular but possibly misleading since the relation between $\bar{d}$ and $D$ is not generally known.

A direct comparison of (2.3) with numerical results is tentative because the spherical cap bubble limit is beyond the capabilities of the numerical methods which have been applied to the problem. The calculations of Ryskin \& Leal (1984) seem reliable and they work well for Weber and Reynolds numbers at which the spheres are greatly distorted. Their figure 1 is a plot of $\bar{C}_{D}$ vs. $\bar{W}$ with $\bar{R}_{e}$ as a parameter, which is in agreement with (2.2) for $\bar{R}_{e}<20$. The empirical formula could not be tested for the large values of $W$ for which the cap bubbles arise but the $\bar{R}_{e}$ variation for smaller values of $\bar{W}$ is also consistent with (2.2).

The comparison of theory and experiment for the case when the deviation $s$ from sphericity is not zero is complicated. In this case the curvature of the nose of the bubble is different from the spherical radius $R$ used in our calculations and those of Davies \& Taylor. We have already noted that the analysis leading to $s$ neglects some of the changes in the potential function which arise from the change in the shape of the domain. The computation or measurement of $s$ will not be undertaken here but the comparison mode in figures 1 and 2 of the paper by Miksis et al. (1982) for distorted bubbles in lenticular shape, but far from spherical caps, may point the way.

\section{Conclusions}

Viscous potential flow is as a potential flow solution of the Navier-Stokes equations in which the vorticity vanishes and no-slip conditions at the interface are not enforced. This solution does not require that the viscosity be put to zero and it is not a good idea to put it to zero. Using this theory, we extended the analysis of Davies \& Taylor (1950) of the spherical cap bubble to include effects of viscosity, surface tension and the deviation of the bubble nose from sphericity. The result of these analyses are then expressed by the rise velocity formula (1.22) and the drag formula (2.3). These formulae are in good agreement with experiments of Bhaga \& Weber (1981) at the high Morton numbers for which the cap bubbles arise, with the caveat that the conversion of the spherical radius to an effective volume equivalent radius is ambiguous. The possible effects of vorticity boundary layers on the rise velocity have not been analysed here; it is not possible to use the same methods that work for spherical gas bubbles and it is unlikely that the results of such an analysis would greatly improve the agreement between theory and experiments documented here.

This work was supported in part by the DOE (engineering research program of the Dept. of Basic Engineering Sciences) and the NSF under grants from Chemical Transport Systems.

\section{REFERENCES}

Batchelor, G. K. 1967 An Introduction to Fluid Dynamics, pp. 475-476. Cambridge University Press. BAtChelor, G. K. 1987 The stability of a large gas bubble rising through liquid. J. Fluid Mech. 184, 399-422.

Bhaga, T. \& Webber, M. 1981 Bubbles in viscous liquids: shapes, wakes and velocities. J. Fluid Mech. 105, 61-85. 
Boulton-Stone, J. M., Robinson, P. B. \& Blake, J. R. 1995 A note on the axisymmetric interaction of pairs of rising, deforming gas bubbles. Intl J. Multiphase Flow 21, 1237-1241.

Davies, R. M. \& TAYLOR, G. I. 1950 The mechanics of large bubbles rising through liquids in tubes. Proc. R. Soc. Lond. A 200, 375-390.

Funada, T. \& JosePh, D. D. 2001 Viscous potential flow analysis of Kelvin-Helmholtz instability in a channel. J. Fluid Mech. 445, 263-283.

FunAda, T. \& JosePH, D. D. 2002 Viscous potential flow analysis of capillary instability. Intl J. Multiphase Flow 28, 1459-1478.

Grace, J. R., Wairegi, T. \& Brophy, J. 1978 Break-up of drops and bubbles in stagnant media. Can. J. Chem. Engng 65, 3-8.

Haberman, W. L. \& Morton, R. K. 1953 An experimental investigation of the drag and shape of air bubbles rising in various liquids. US (Navy) David W. Taylor Model Basin Rep. 802.

Harper, J. J. 1972 The motion of bubbles and drops through liquids. Adv. Appl. Mech. 12, 59-129.

Hesla, T., Huang, A. \& Joseph, D. D. 1993 A note on the net force and moment in a drop due to surfaces forces. J. Colloid Interface Sci. 158, 255-257.

Joseph, D. D., Beavers, G. S. \& Funada, T. 2002 Rayleigh-Taylor instability of viscoelastic drops at high Weber numbers. J. Fluid Mech. 453, 109-132.

Joseph, D. D., Belanger, J. \& Beavers, G. S. 1999 Breakup of a liquid drop suddenly exposed to a high-speed airstream. Intl J. Multiphase Flow 25, 1263-1303.

JosePh, D. D. \& Liao, T. Y. 1994 Potential flow of viscous and viscoelastic fluids. J. Fluid Mech. 265, 1-23.

Kang, I. S. \& LEAL, L. G. 1988 The drag coefficient for a spherical bubble in a uniform streaming flow. Phys. Fluids 31, 233-237.

Levich, V. G. 1949 The motion of bubbles at high Reynolds numbers. Zh. Eksp. Teor. Fiz. 19, 18; see also Physiochemical Hydrodynamics, English translation by Scripta Technica, Prentice-Hall, Englewood Cliffs, NJ, 1962, p. 436ff.

Miksis, M., Vanden-Broeck, J.-M. \& Keller, J. B. 1982 Rising bubbles. J. Fluid Mech. 123, 31-41.

Moore, D. W. 1959 The rise of a gas bubble in a viscous liquid. J. Fluid Mech. 6, 113-130.

Moore, D. W. 1963 The boundary layer on a spherical gas bubble. J. Fluid Mech. 16, 161-176.

Ryskin, G. \& LEAL, L. G. 1984 Numerical solution of free-boundary problems in fluid mechanics. Part 1. The finite-difference technique. J. Fluid Mech. 148, 19-35.

TAYLOR, T. D. \& ACRIVOS, A. 1964 On the deformation and drag of a falling viscous drop at low Reynolds number. J. Fluid Mech. 18, 466-476.

Vania, F., Pardo, R., Yänez, R., Trallero, J. \& JosePh, D. D. 2002 Universal correlation for the rise velocity of long gas bubbles in round pipes. J. Fluid Mech., accepted, also available at http://www.aem.umn.edu/people/faculty/joseph/ViscousPotentialFlow/.

Wallis, G. B. 1969 One-dimensional Two-phase Flow. McGraw-Hill.

Wegener, P. P. \& Parlange, J.-Y. 1973 Spherical-cap bubbles. Annu. Rev. Fluid Mech. 5, 79-100. 